and D. George (eds.)

\title{
Opheliidae (Polychaeta) collected by the R/V Hero and the USNS Eltanin cruises from the Southern Ocean and South America
}

\author{
NANCY J. MACIOLEK and JAMES A. BLAKE \\ ENSR Marine \& Coastal Center, 89 Water Street, Woods Hole, Massachusetts 02543, USA \\ E-mail: njmaciolek@gmail.com
}

\begin{abstract}
SUMMARY: Opheliid polychaetes collected by the United States Antarctic Research Program and additional material from South America were made available for study through the Smithsonian Institution, Washington, D.C., USA. The Opheliidae from Antarctic seas were treated by Hartman $(1966,1967,1978)$, who summarized earlier work and also described several new species. Of the five genera she recorded, two (Travisia and Kesun) have since been synonymized and one, Ammotrypane, has been referred to Ophelina; Hartman's records therefore encompass four genera, three of which are represented in the present collections. No representatives of Euzonus (Thoracophelia) were found. The following species were recorded from Antarctica in the present study: Ophelia algida n. sp., Ophelina breviata (Ehlers, 1913), Ophelina cf. cylindricaudata (sensu Hartmann-Schröder and Rosenfeldt, 1989), Ophelina nematoides (Ehlers, 1913), Ophelina scaphigera (Ehlers, 1900), Ophelina cf. setigera (Hartman, 1978), Ophelina syringopyge (Ehlers, 1901), Travisia antarctica Hartman, 1967 (emended 1978), Travisia kerguelensis McIntosh, 1885, Travisia kerguelensis gravieri Monro, 1930, Travisia palmeri n. sp. and Travisia tincta n. sp. The new species of Ophelia is distinguished by having branchiae starting on chaetiger 2 (rather than on or after chaetiger 8 as in most species of this genus). Travisia palmeri has 22 chaetigers and is distinguished by long branchiae. Travisia kerguelensis gravieri is raised to full species status and renamed T. monroi. Travisia tincta $\mathrm{n}$. sp., distinguished by a distinctive Methyl Green staining pattern and long branchiae, is described from off Peru.
\end{abstract}

Keywords: Antarctica, Peru, Opheliidae, Ophelia, Ophelina, Travisia, systematics, polychaete.

RESUMEN: OPHELIIDAE (POLYCHAETA) RECOLECTADOS POR EL BIO HERO Y LAS CAMPAÑAS DEL USNS ELTANIN EN LOS OCÉANOS MERIDIONALES Y SUDAMÉRICA. - Los poliquetos ofélidos objeto del presente estudio corresponden al material recolectado por el Programa de Investigación Antártico de los Estados Unidos así como material adicional procedente de Sudamérica. Este material ha sido puestos a disposición de los autores por la Institución Smithsoniana, Washington, D.C., Estados Unidos de América. Los Opheliidae de los mares antárticos fueron previamente estudiados por Hartman (1966, 1967, 1978), quien reunió lo publicado hasta el momento y describió nuevas especies. De los cinco géneros registrados por ella, dos (Travisia y Kesun) han sido desde entonces sinonimizados y uno, Ammotrypane, ha sido incluido en Ophelina. Así, los registros de Hartman incluyen cuatro géneros, tres de los cuales están representados en las colecciones estudiadas. No se han encontrados representantes de Euzonus (Thoracophelia). En el presente estudio se registran las siguientes especies de la Antártida: Ophelia algida n. sp., Ophelina breviata (Ehlers, 1913), Ophelina cf. cylindricaudata (sensu Hartmann-Schröder and Rosenfeldt, 1989), Ophelina nematoides (Ehlers, 1913), Ophelina scaphigera (Ehlers, 1900), Ophelina cf. setigera (Hartman, 1978), Ophelina syringopyge (Ehlers, 1901), Travisia antarctia Hartman, 1967 (enmendada 1978), Travisia kerguelensis McIntosh, 1885, Travisia kerguelensis gravieri Monro, 1930, Travisia palmeri $\mathrm{n}$. sp. y Travisia tincta $\mathrm{n}$. sp. La nueva especie de Ophelia se caracteriza por presentar bránquias a partir del chaetígero 2 (y no a partir del chaetigero 8-11 como ocurre en la mayoria de las especies de este género). Travisia palmeri posee 22 chaetigeros y se caracteriza por sus largas bránquias. Travisia kerguelensis gravieri se eleva a rango de especie y se renombra como T. monroi. Travisia tincta n. sp., caracterizada por sus largas bránquias y un peculiar patrón al ser teñida, se describe de las costas de Perú.

Palabras clave: Antártida, Perú, Opheliidae, Ophelia, Ophelina, Travisia, sistemática, poliquetos, ofélidos. 


\section{INTRODUCTION}

Collections of polychaetes taken by the United States Antarctic Research Program (USARP) between 1969 and 1982, and additional material collected from off the coast of Peru as part of the Southeastern Pacific Biological and Oceanographic Program (SEPBOP), were made available for study through the Smithsonian Institution, Washington, D.C., USA. Treatment of these materials has been focused at the family level; the present paper covers the Opheliidae in these collections.

The known species of Opheliidae from Antarctic seas were studied by Hartman (1966, 1967, 1978), who mainly summarized work by Ehlers, Kinberg, and Monro, and also described several new species. Of the five genera Hartman recorded, two (Travisia and Kesun) have since been synonymized and one, Ammotrypane, has been referred to Ophelina; thus Hartman's records encompass four genera, three of which are represented in the present collections. Hartmann-Schröder and Rosenfeldt (1989, 1991) examined collections made in the Antarctic by German research expeditions and recorded two species of Travisia and five species of Ophelina. Opheliids from South American areas have been better studied, most recently by Elias and Bremec (2003) from Argentina and Elias et al. (2003) from Brazil.

The following species and genera are included in this paper:

Ophelia algida $\mathrm{n} . \mathrm{sp}$.

Ophelina breviata (Ehlers, 1913)

Ophelina cf. cylindricaudata (Hansen,1878)

sensu Hartmann-Schröder and Rosenfeldt, 1989

Ophelina nematoides (Ehlers, 1913)

Ophelina scaphigera (Ehlers, 1900)

Ophelina cf. setigera (Hartman, 1978)

Ophelina syringopyge (Ehlers, 1901)

Travisia antarctica Hartman, 1967 (emended 1978)

Travisia kerguelensis McIntosh, 1885

Travisia monroi, new name for Travisia

kerguelensis gravieri Monro, 1930

Travisia tincta $\mathrm{n}$. sp.

Travisia palmeri $\mathrm{n} . \mathrm{sp}$.

\section{SYSTEMATIC ACCOUNT}

Family OPHELIIDAE Malmgren, 1867

Type genus. Ophelia bicornis Savigny, 1818
Diagnosis. Body cylindrical, tapered anteriorly and truncated posteriorly, with up to 60 segments; segments similar, body not divided into distinct regions. Body either (a) uniformly slender or slightly inflated anteriorly, with midventral and lateral grooves from chaetiger 1 or 7 , or (b) short, thick, grub-shaped, lacking ventral groove. Body surface may be smooth or subdivided into annuli (or areolations). Prostomium small, tapered, blunt or conical, sometimes ending in a slender palpode; pair of evaginable nuchal organs present, other appendages lacking; subdermal eyes present or absent. Peristomium fused with prostomium and first chaetiger; mouth a ventral transverse slit at level of first chaetiger. Pharynx large, eversible, unarmed, saclike, and may be lobed or cushion-like.

Parapodia biramous, rami small, button-shaped lobes; dorsal cirri lacking; short ventral cirri present or absent. Chaetae all simple capillaries. Branchiae present or absent; if present, cirriform or branched; branchiae present along majority of body, confined to midregion, or confined to anterior and posterior, lacking in midregion. Interramal sensory organs may be present as small eyespots, sensory pits, or papillae. A few preanal segments may be achaetous, telescoped, and/or retractile. Anus terminal; pygidium often prolonged and tubular, ending with circlet of blunt lobes, or two stout ventral lobes and a circlet of dorsolateral papillae, or with papillate tube and long internal cirrus.

Remarks. The opheliids are active burrowers and bottom deposit feeders. They have separate sexes, with spawning taking place in or on the bottom surface; however, some forms become pelagic as sexually mature epitokes. The larvae are planktonic and settle at about the 5-chaetiger stage. Opheliids are well known for their specific distribution patterns, being restricted to substrata with a relatively narrow range of particle size. Some species are characteristic of fine muds, others of relatively coarse sands; some are found at shallow depths, others in deep water.

Currently, nine genera in three subfamilies are recognized (Dauvin and Bellan, 1994). The three subfamilies-Opheliinae, Ophelininae, and Travisiinae-were established by HartmannSchröder (1971). The Travisiinae appear to be closely related to the Scalibregmatidae in terms of body form and appearance, whereas the other two have a long slender appearance. Blake (2000) and Rouse 
(2001) suggested that Travisia Johnston had a close affinity with the scalibregmatids, and a recent cladistic analysis by Persson and Pleijel (2005), based on results of 18S rDNA and 28S rDNA analysis, showed Travisia clearly nested within the scalibregmatids.

\section{Genus Ophelia Savigny, 1818}

\section{Type species. Ophelia bicornis Savigny, 1818}

Diagnosis (emended). Body divided externally into two regions: an anterior cylindrical region and a posterior region with a deep ventral and two lateral grooves. Prostomium a small pointed cone, lacking appendages; pair of nuchal organs on posterior lateral border. Branchiae present or absent; branchiae, if present, cirriform with single or, rarely, double filaments, present from chaetigers 2 or 8-11, absent from a few posterior preanal segments. Chaetae all simple capillaries; parapodia low rounded lobes. Lateral sensory organs present between parapodial rami. Anal segment with papillae.

Remarks. Tebble (1953) established diagnostic characteristics for the genus Ophelia. Among these, he concluded that the most important were the number of branchiate and abranchiate chaetigers, the presence or absence, and extent if present, of branchial fenestrations (small pores in the lateral groove), and the structure of the posterior region, including the development of dorsal ridges vs telescoping of the segments. At the time of Tebble's work, all of the described Ophelia species had branchiae; however, four abranchiate species have subsequently been described, and the number of valid species in the genus is at least 32 (Bellan and Dauvin, 1991). The present emendation takes into account the presence of branchiae from chaetiger 2 in the new species described below.

Hartman (1966) reported two species of Ophelia from the Antarctic: $O$. bipartita Monro, 1936 and $O$. praetiosa (Kinberg, 1866), but neither species was encountered in this study. The new species described below was represented by a single specimen from subantarctic Macquarie Island. Three other small and poorly preserved Ophelia specimens were also present: the gut of one of those small specimens contained exceptionally large sand particles and biological remnants, causing one to wonder at the elasticity of the opheliid body.
Ophelia algida, n. sp.

(Fig. 1)

Material examined. R/V Eltanin Cruise 27, Sta. 1974, 15 Feb 1967, $54^{\circ} 30^{\prime} \mathrm{S}-54^{\circ} 34^{\prime} \mathrm{S}, 158^{\circ} 59^{\prime} \mathrm{E}, 112-124 \mathrm{~m}$, 1 specimen (Holotype, USNM 1078733).

Description. Specimen complete, $10 \mathrm{~mm}$ long and $0.75 \mathrm{~mm}$ wide for 35 chaetigers. Prostomium bluntly rounded, with large palpode at tip; two welldeveloped nuchal organs present at base of prostomium (Fig. 1). Segments pentannulate. Ventral groove well defined from chaetiger 7 (with faint longitudinal line on first six chaetigers); lateral grooves also from chaetiger 7 , parapodia recessed in lateral grooves.

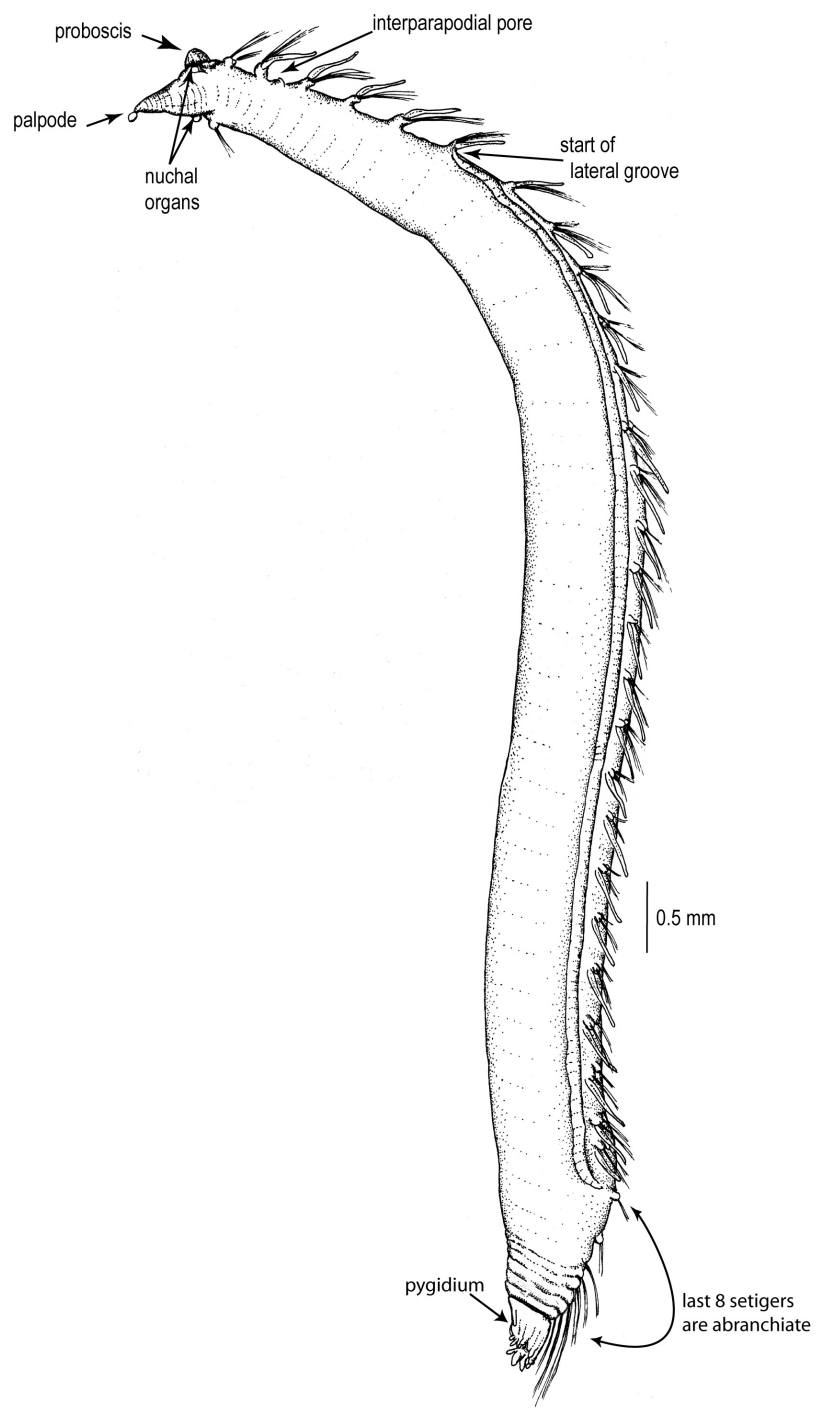

FIG. 1. - Ophelia algida n. sp. (Holotype, R/V Eltanin Cruise 27, Sta. 1974, 15 Feb 1967, USNM 1078733). Entire animal in lateral view. 
Branchiae from chaetiger 2, continue throughout body, absent from last eight preanal chaetigers (branchial formula $1 \mathrm{a}+26 \mathrm{~b}+8 \mathrm{a}=35$ ); branchiae slender, cirriform, not especially long. Branchial fenestrations not seen except for one interparapodial pore between chaetigers 2 and 3 .

Parapodia small, broadly rounded, with slightly pointed tip in some chaetigers. Chaetae all delicate golden capillaries in two fascicles, one arising from dorsal side of parapodial lobe, second emerging from base of ventral side of parapodial lobe. Notochaetae long, especially in anterior chaetigers where they arch over dorsum and nearly meet at midline; chaetae of last three or four preanal chaetigers also exceptionally long and form a delicate cage around the anal structure; anal segment itself achaetous. Posterior segments without dorsal longitudinal ridges, these segments slightly telescoped dorsally, pulling (tilting) the anal structure towards the dorsum (Fig. 1). Pygidium with about 16 papillae (processes), ventral papillae only slightly longer than dorsal ones.

Methyl Green staining pattern. Two patches of deep stain on ventral surface of pygidium, otherwise stain not retained.

Etymology. The species name is taken from the Latin algidus, meaning cold, and refers to the first collection of this species from the cold waters of the Southern Ocean.

Remarks. This species appears to combine features from two genera: the origin of the deep ventral groove on chaetiger 7 and the structure of the pygidium, with the associated long capillary chaetae, place this species in the genus Ophelia. However, the shape of the prostomium with a terminal palpode and the origin of the branchiae from chaetiger 2 associate the species with the genus Ophelina. Only one interramal papilla (lateral sense organ), between chaetigers 2 and 3 on one side of the specimen, was noted. The bundles of chaetae on each chaetiger are not widely separated as in many species of Ophelia (i.e. there is only one parapodial lobe with two bundles of chaetae arising from it) giving the appearance of a uniramous rather than biramous condition.

Distribution. 112 m, Southern Ocean near Macquarie Island.

\section{Genus Ophelina Oersted, 1843}

Ophelina Oersted, 1843. Type species: Ophelina acuminata Oersted, 1843.

Ammotrypane Rathke, 1843. Type species: Ammotrypane aulogaster Rathke, 1843. Fide Støp-Bowitz, 1945.

Ladice Kinberg, 1866. Fide Fauchald, 1977.

Omaria Grube, 1869. Fide Fauchald, 1977.

Terpsichore Kinberg, 1866. Fide Fauchald, 1977.

Urosiphon Chamberlin, 1919. Fide Fauchald, 1977.

Diagnosis. Body elongate, not divided into distinct regions; with deep ventral groove and two lateral grooves along entire length of body. Prostomium conical, sometimes with terminal palpode; eyes present or absent. Branchiae present or absent; if present, beginning on chaetiger 2 , continuing to posterior end, sometimes absent from middle or far posterior chaetigers; branchiae single, cirriform. Segmental lateral eyes absent. Noto- and neuropodia with small fascicles of capillary chaetae; small ventral cirrus present. Pygidium with anal funnel sometimes bearing long unpaired cirrus and additional lateral cirri.

Remarks. Chamberlin (1919:385) pointed out that the type of Ophelina Oersted (1843), O. acuminata Oersted, is identical with the type of Ammotrypane H. Rathke (1843), A. aulogaster H. Rathke. Chamberlin used Ammotrypane as the valid genus, as did Hartman (e.g. 1967) and other authors. However, Støp-Bowitz (1945) noted that Marenzeller (1892) determined that Oersted's work had been published before Rathke's, and therefore Ammotrypane was the junior synonym of Ophelina. Hartmann-Schröder (1971) and Fauchald (1977) recognized Ophelina as the valid name for the genus, and subsequent authors have followed this version of the synonymy.

The anal funnel is a highly diagnostic feature of Ophelina species, but it is often broken off, especially if specimens are handled roughly during sampling and preservation. Species that are described as lacking the anal funnel are most likely based on specimens where this structure has been lost. Following this observation, Blake (2000), in treating Ophelina species from California, referred O. pallidus (Hartman, 1960) to synonymy with $O$. acuminata Oersted, 1843. The Antarctic species $O$. gymnopyge (Ehlers, 1908), also described as lacking an anal funnel, is therefore similarly questionable, and records of that species (Hartman, 1966; Hartmann-Schröder and Rosenfeldt, 1989, 1991; 
Elias et al., 2003) should be treated with caution as they most likely are damaged specimens of other species.

Blake (2000) pointed out that although the number of chaetigers is fixed in certain opheliid species, this condition does not appear to pertain to Ophelina acuminata, a common, perhaps cosmopolitan opheliid, in which segments are added with continued growth; he concluded that the number of chaetigers is not an important characteristic in this genus.

Hartman $(1966,1967,1978)$ included six named species of Ophelina (as Ammotrypane) from Antarctic waters: Ophelina breviata (Ehlers, 1913), O. gymnopyge (Ehlers, 1908), O. nematoides (Ehlers, 1913), O. scaphigera (Ehlers, 1900), O. setigera Hartman, 1978, and O. syringopyge (Ehlers, 1901). She also suggested that two additional species might be present in her collections: a branchiate species having nine bifid papillae on the anal funnel, and an abranchiate species that cooccurred with $O$. nematoides. Hartmann-Schröder and Rosenfeldt (1989) reported $O$. cylindricaudata (Hansen, 1878) as a new record for the Antarctic; this species was also reported by Hilbig (2001) and Siciński (2004).

Ophelina was the most common genus in the Antarctic samples, and was represented by six species: O. breviata (Ehlers, 1913), O. cf. cylindricaudata (Hansen, 1878) (sensu HartmannSchröder and Rosenfeldt, 1989), O. nematoides (Ehlers, 1913), O. scaphigera (Ehlers, 1900), O. cf. setigera (Hartman, 1978), and O. syringopyge (Ehlers, 1901), which was the most common species recorded.

Ophelina breviata (Ehlers, 1913)

Ammotrypane breviata Ehlers, 1913, pp. 523-524. Monro, 1930, p.
165. Monro, 1936, p. 165. Monro, 1939, pp. 133-134. Hartman,
1953, p. 48. Hartman, 1966, p. 47, Plate XV, figs. 1-3.
Hartman, 1978, p. 183 (in part?). Ophelina breviata Augener, 1932, p. 42.

Material examined. R/V Eltanin Cruise 6, Sta. 412, 62 06'$62^{\circ} 06^{\prime} \mathrm{S}, 56^{\circ} 00^{\prime}-55^{\circ} 59^{\prime} \mathrm{W}, 01$ Jan 1963 , Menzies trawl, ca. $1180 \mathrm{~m}$, 7 specimens (USNM 1078699); Cruise 9, Sta. 693, 56 53'-56 $54^{\circ}$ ' S, 3731' $-37^{\circ} 32^{\prime} \mathrm{W}, 28$ Aug 1963 , Menzies trawl, $3138 \mathrm{~m}, 1$ specimen (USNM 1078700); Cruise 51, Sta. 5762, 76 $02.1^{\prime}-76^{\circ} 02.3^{\prime} \mathrm{S}$, $179^{\circ} 57.0-179^{\circ} 52.1^{\prime} \mathrm{W}, 09 \mathrm{Feb} 1972$, Menzies trawl, 347-358 m, 1 specimen (USNM 1078703); R/V Hero Cruise 692, Sta. 402, $53^{\circ} 41^{\prime}-53^{\circ} 38^{\prime} \mathrm{S}, 70^{\circ} 24^{\prime}-70^{\circ} 24^{\prime} \mathrm{W}, 13$ May $1969,90-110 \mathrm{~m}, 3$ specimens (USNM 187912); Sta. 18-1, 64 $18.55^{\prime}-64^{\circ} 19.00^{\prime} \mathrm{S}$ $62^{\circ} 58.67-62^{\circ} 58.00^{\prime} \mathrm{W}, 22$ Mar 1982, Blake trawl, 2 specimens (USNM 187907); Sta. 22-1, 642․ $22.30-64^{\circ} 22.10^{\prime}$ 'S, $61^{\circ} 28.3-$ 61 ${ }^{\circ} 29.30^{\prime}$ 'S, 23 Mar 1982, 60-152 m, 1 specimen (USNM 187911); Sta. 41-1, 64 $48.42^{\prime} \mathrm{S}, 64^{\circ} 07.00^{\prime} \mathrm{W}, 29$ Mar 1982, Blake trawl, $88^{-}$ $112 \mathrm{~m}, 1$ specimen (USNM 1078712); Cruise 824, Sta. 9-1, $66^{\circ} 08.1^{\prime} \mathrm{S}, 66^{\circ} 35.5^{\prime} \mathrm{W}, 18$ Mar 1982 , dredge, $140-210 \mathrm{~m}, 1$ speci- men (USNM 187910); Cruise 833, Sta. 18-1, 62 $41.5^{\prime} \mathrm{S}, 56^{\circ} 01.75^{\prime}$, W, 01Apr 1983, Blake trawl, 210-240 m, 1 specimen (USNM 187908).

Diagnosis. Body long, linear, 17-34 mm long, up to $2 \mathrm{~mm}$ wide; segments number 24-28; body with longitudinal ventral groove; last four chaetigers characteristically short and crowded, pushed towards mid-ventral line. Prostomium conical, longer than wide, terminating in a small palpode; with pair of nuchal organs at base of prostomium. Branchiae cirriform, on all chaetigers except first one and last four. Anal cylinder long, smooth or faintly and irregularly ringed, with the dorsal edge slightly longer than the ventral edge, ending in several small indistinct lobes.

Methyl Green staining pattern. No discernable pattern.

Remarks. Ophelina breviata has branchiae present on all chaetigers except the first and the last four, but Hartman (1978) noted that her material from the Weddell Sea and Hero Inlet lacked branchiae on the majority of middle body chaetigers, perhaps because of damage. The absence of branchiae from the middle region suggests that some of Hartman's material may represent a species distinct from $O$. breviata; perhaps $O$. cylindricaudata (Hansen, 1878), as reported by Hartmann-Schröder and Rosenfeldt (1989) as a new record for the Antarctic.

$O$. breviata was recently recorded in deep water on the continental slope off the Farallon Islands in northern California (Maciolek and Blake, unpublished data).

Distribution. Antarctic waters, 20-385 m (Wilhelm II coast, 385 m; off Signy Is., 244-344 m; 141-219 m; Enderby land, 219-220 m; South Georgia, $20 \mathrm{~m}$; Peter I Island, 70-96 m); northern California, $3000 \mathrm{~m}$.

Ophelina cf. cylindricaudata (Hansen, 1878) (sensu Hartmann-Schröder and Rosenfeldt, 1989)

Ophelina cylindricaudata (Hansen, 1878). Hartmann-Schröder and Rosenfeldt, 1989, p. 74, figs 14-16. Hartmann-Schröder and Rosenfeldt, 1991, p.76.

Material examined. R/V Eltanin Cruise 21, Sta. 272, 60²1'6024'S, $120^{\circ} 03^{\circ}-120^{\circ} 09^{\prime} \mathrm{W}, 26$ Dec 1965, 600-1000 m, 1 specimen (USNM 1078701); R/V Hero Cruise 721, Sta. 824, 64¹9.6'S, $62^{\circ} 58.8^{\prime} \mathrm{W}, 18$ Jan 1972 , Blake trawl, $156 \mathrm{~m}, 1$ specimen (USNM 1078704); Sta. 1033, 64 $13.0^{\prime} \mathrm{S}, 61^{\circ} 05.4^{\prime} \mathrm{W}, 16 \mathrm{Dec} 1971$, Petersen grab, 129 m, 3 specimens (USNM 1078705); Sta. 1035, 64¹3.0'S, 
6105.4'W, 16 Dec 1971, Petersen grab, $118 \mathrm{~m}, 1$ specimen

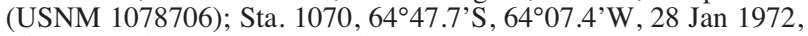
Blake trawl, $100 \mathrm{~m}, 1$ specimen (USNM 1078707); Sta. 1130,

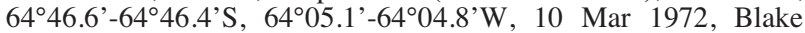
trawl, 65-75 m, 2 specimens (USNM 1078708); Cruise 731, Sta.

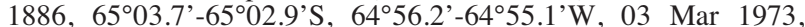
Blake trawl, 300-410 m, 1 specimen (USNM 187909); Sta. 1937,

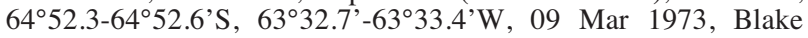
trawl, 96-113 m, 1 specimen (USNM 1078710); Cruise 824, Sta. 5-

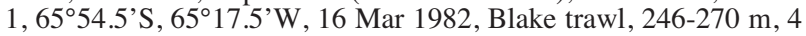
specimens (USNM 1078711); Sta. 18-1, 64 $18.55^{\prime}-64^{\circ} 19.00^{\prime}$ 'S, $62^{\circ} 58.67-62^{\circ} 58.00^{\prime} \mathrm{W}, 22$ Mar 1982, Blake trawl, 1 specimen

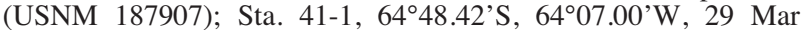
1982, Blake trawl, 88-112 m, 2 specimens (USNM 1078712).

Diagnosis. Body long, linear, 17-34 mm long, up to $2 \mathrm{~mm}$ wide; segments number 24-28; body with longitudinal ventral groove; last four chaetigers characteristically short and crowded, pushed towards median ventral line. Prostomium conical, longer than wide, with rounded edge, palpode absent; with pair of large, well-developed nuchal organs at base of prostomium. Branchiae cirriform, present on chaetigers 2-9, absent from mid-body region, present on last six of 10 chaetigers, last four crowded chaetigers without branchiae. Anal cylinder long, smooth or faintly and irregularly ringed, with the dorsal edge obviously longer than the ventral edge; ventral filament of anal tube, subtriangular, wider at base, tapering to narrow tip, wrinkled in appearance.

Methyl Green staining pattern. No discernable pattern.

Remarks. Hansen (1878) described Ophelina cylindricaudata (as Ammotrypane cylindricaudatus) from two specimens collected in 763 and $911 \mathrm{~m}$ off the coast of Norway. The species was described as having a long anal funnel ending with a dorsal papilla overhanging a shorter, wider ventral lip (see Hansen, plate VI, fig. 3). Neither Hansen (1878) nor Fauvel $(1914,1927)$ mentioned a long ventral filament associated with the anal tube, but this feature appears in descriptions provided by Støp-Bowitz (1945, 1948). Hartmann-Schröder and Rosenfeldt $(1989,1991)$ accepted the description and illustration by Stop-Bowitz (1945), and recorded the species from the Antarctic. However, it is possible that the Antarctic species is not the same as O.cylindricaudata (Hansen), which has been recorded primarily from Arctic waters. An analysis encompassing additional material from both Arctic and Antarctic waters is necessary before a conclusion can be reached regarding the Antarctic material.
Distribution. Fide Hartmann-Schröder (1996) O. cylindricaudata (Hansen) is known from Arctic and Antarctic Seas, 7-4663 m; Antarctic material of $O$. cylindricaudata sensu Hartmann-Schröder and Rosenfeldt (1989) was recorded from ca. 65$600 \mathrm{~m}$.

\section{Ophelina nematoides (Ehlers, 1913)}

Ammotrypane nematoides Ehlers 1913, pp. 524-525. Hartman, 1966, p. 49. Hartman, 1978, p. 183

Material examined. R/V Eltanin, Cr. 6, Sta. $412,62^{\circ} 06^{\prime}$ S, $56^{\circ} 00^{\prime}-$ $55^{\circ} 59^{\prime} \mathrm{W}, 1$ Jan 1963, Menzies trawl, $1180 \mathrm{~m}, 1$ specimen (incomplete, partly dried out) (USNM 187903); R/V Hero, Cruise 824, Sta. 5-1, 1 specimen (USNM 187913); R/V Glacier, Cruise 2, Sta. 4, $77^{\circ} 05.5^{\prime} \mathrm{S}, 35^{\circ} 04^{\prime} \mathrm{W}, 793 \mathrm{~m}$, anchor dredge, 26 Feb 1969, 2 incomplete specimens (USNM 1078713), Cruise 2, Sta. 5, $77^{\circ} 19.8^{\prime} \mathrm{S}$, 36²1.3'W, 1079 m, 27 Feb 1969, 2 incomplete specimens (USNM 1078714).

Diagnosis. Body thin, threadlike, resembling a nematode. Reported length $30 \mathrm{~mm}$, width $1 \mathrm{~mm}$ for 30 chaetigers; present material $4 \mathrm{~mm}$ and $0.2 \mathrm{~mm}$ wide for ca.18 chaetigers, not including anal funnel, which is missing on all specimens. Prostomium bluntly conical, about as long as first 2 segments, terminating anteriorly in a palpode. Ventrum with longitudinal medial groove; lateral grooves also present. Segments not annulated. Parapodia small, rounded. Branchiae absent. Pygidium (described) with an annulated cylindrical postanal cylinder, about as long as last two segments.

Methyl Green staining pattern. No particular staining pattern; stain is taken up uniformly except for the very tip of the prostomium and posterior end, which do not stain. Ventral groove does not appear as stripe as in $O$. syringopyge.

Remarks. In Hartman's (1978) description of the species, she mentions a short slender median antenna inserted slightly behind the tip of the prostomium, but she is undoubtedly referring to the palpode at the anterior edge.

Distribution. Antarctica, 246-2725 m.

Ophelina scaphigera (Ehlers, 1900)

Ammotrypane scaphigera Ehlers, 1900b, p. 218. Ehlers, 1901, p. 172, pl. 22, figs. 1-4. Monro, 1936, p. 165. Hartman, 1953, p. 48. Hartman, 1966, p. 49, pl. XV, figs.7-9.

Material examined. R/V Eltanin, Cruise 7, Sta. 475, 57 $11^{\prime}$ ' $57^{\circ} 14^{\prime} \mathrm{S}, 4^{\circ} 53^{\prime}-44^{\circ} 47^{\prime} \mathrm{W}, 3382 \mathrm{~m}$, Menzies trawl, 14 Feb 1963, 1 specimen (USNM 1078716). 
Diagnosis. Present specimen with 32 chaetigers, measuring $1 \mathrm{~mm}$ wide, $35 \mathrm{~mm}$ long including anal funnel; other material described as up to $21 \mathrm{~mm}$ long for 39 segments. Prostomium conical, terminating in large palpode; pair of large nuchal organs at base of prostomium. Branchiae present from chaetiger 2, absent from last six chaetigers. Anal end scoop-shaped, with a ventral concavity and a pair of large cirriform processes inserted ventrally; long, slender annulated filament extends beyond end of funnel.

Methyl Green staining pattern. The large palpode on the prostomium and the everted nuchal organs were the only two structures that did not retain stain.

Remarks. The distinctive anal funnel on this species is remarkably similar to that found on the widespread species Ophelina acuminata. Elias et al. (2003) report $O$. cf. acuminata from 33-71 m off SE Brazil, but their material has branchiae on all chaetigers, except the first. They did not consider $O$. scaphigera. However, until such time as Ehlers types can be examined and compared with $O$. acuminata, no further conclusions can be drawn. The present record extends the depth range of this species from $385 \mathrm{~m}$ to $3382 \mathrm{~m}$.

Distribution. Antarctica, 20-3382 m.

\section{Ophelina cf. setigera (Hartman, 1978)}

Ammotrypane setigera (Hartman, 1978) pp. 183-184, fig 30.

Material examined. R/V Eltanin Cruise 32, Sta. 2024, $75^{\circ} 23^{\prime}$ S, $174^{\circ} 11^{\prime} \mathrm{W}, 16$ Jan 1968 , camera grab, $1253 \mathrm{~m}, 1$ specimen (USNM 1078702).

Diagnosis. Large species, measuring up to 12 $\mathrm{mm}$ wide and $26 \mathrm{~mm}$ long for 28 chaetigers Body with longitudinal ventral groove; last four chaetigers short and crowded, pushed towards median ventral line. Prostomium oval, bluntly rounded on anterior margin, with very small anterior protuberance (palpode); pair of large nuchal organs at base of prostomium. Parapodia with fleshy lobes; chaetae all capillaries, very long anteriorly, shorter posteriorly. Branchiae cirriform, on all chaetigers except first one and last four. Chaetigers appear multiannulate. Anal funnel cylindrical, longest middorsally, terminating in 12-14 short digitate processes (anal funnel lost in present material).
Methyl Green staining pattern. No particular pattern.

Remarks. Hartman (1978) distinguished O. setigera by the long chaetae in the first few chaetigers; in addition, the multiannulate appearance of the chaetigers and the gradual diminishing in size of the last 16 (of 43) chaetigers appear to be specific characteristics. The present material differs from Hartman's description in that although the animals are much larger (260 $\mathrm{mm}$ vs $40 \mathrm{~mm}$ long), there are fewer chaetigers (28 vs 43). Also, the posterior chaetigers do not diminish in size but remain essentially equal throughout, with the exception of the last four, which are characteristically short and crowded together.

Distribution. Weddell Sea, 1664-4757 m; Ross Sea, $1253 \mathrm{~m}$.

\section{Ophelina syringopyge (Ehlers, 1901)}

Ammotrypane aulopyge Ehlers, 1897 pp. 100-121 pl. 6, figs. 157, 158. Ehlers, 1900 a p. 13.

Ammotrypane syringopyge Ehlers, 1901 pp. 171-172. Ehlers, 1913 p. 523. Fauvel, 1936 pp. 32-33. Fauvel, 1951 p. 767. Hartman, 1966 p. 51, pl. XVI. Elias et al., 2003 p. 80.

Material examined. Balleny Island, south end of Buckle Island, 66 53 'S, $163^{\circ} 19^{\prime} \mathrm{E}, 10 \mathrm{Feb} 1974,55-146 \mathrm{~m}$, coll. Scripps, 1 specimen (USNM 187901); Palmer Station, Sta. AH-1, 64'46.63'$64^{\circ} 46.68^{\prime} \mathrm{S}, 64^{\circ} 03.28^{\prime} \mathrm{W}, 15$ Jan $1969,7 \mathrm{~m}, 25$ specimens (USNM 187936); Sta. AH-2, 64 $46.42^{\prime} \mathrm{S}, 6^{\circ} 04.22^{\prime} \mathrm{W}$, grab sample, 17 Jan 1969, 14 specimens (USNM 187930); Sta. AH-3, 6446.22'S,

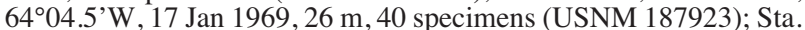

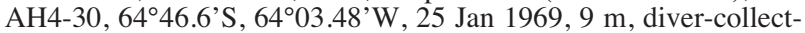
ed, 2 specimens (USNM 187866); R/V Eltanin Cruise 12, Sta. $1003,62^{\circ} 41^{\prime} \mathrm{S}, 54^{\circ} 43^{\prime} \mathrm{W}, 15$ Mar $1964,210-230 \mathrm{~m}, 2$ specimens (USNM 187898); Cruise 32, Sta. 2035, 74³2'S, $168^{\circ} 17^{\prime} \mathrm{E}$, 18 Jan 1968, 876 m, 1 specimen (USNM 187899); R/V Hero Cruise 691, Sta. $26,63^{\circ} 26^{\prime}-63^{\circ} 25^{\prime} \mathrm{S}, 62^{\circ} 15^{\prime}-62^{\circ} 14^{\prime} \mathrm{W}, 5^{\prime}$ Blake trawl, $10 \mathrm{Feb}$ 1969, 119-124 m, 1 specimen (USNM 187867); Cruise 702, Sta.

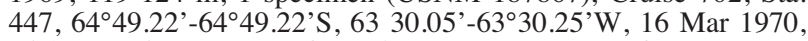
Blake trawl, 20-27 m, 6 specimens (USNM 187868); Sta. 448, $64^{\circ} 69.28^{\prime}-64^{\circ} 49.28^{\prime} \mathrm{S}, 6^{\circ} 30.17^{\prime}-63^{\circ} 30.53^{\prime} \mathrm{W}, 16 \mathrm{Mar} 1970,18-27$ $\mathrm{m}, 13$ specimens (USNM 187869); Cruise 712, Sta. 665, $54^{\circ} 44.85^{\prime} \mathrm{S}, 63^{\circ} 52.9^{\prime} \mathrm{W}, 11$ May 1971, $44 \mathrm{~m}, 2$ specimens (USNM

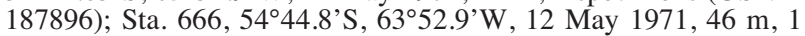
specimen (USNM 187897); Cruise 721, Sta. 703, 62 $16.7^{\prime}$ $62^{\circ} 17.5^{\prime} \mathrm{S}, 58^{\circ} 34.0^{\prime}-58^{\circ} 34.6^{\prime} \mathrm{W}, 21 \mathrm{Dec} 1971,38-74 \mathrm{~m}, 3$ specimens (USNM 187917); Sta. 735, 64 $46.72^{\prime}$ S , 64 ${ }^{\circ} 05.25^{\prime} \mathrm{W}, 31 \mathrm{Dec}$ 1971, Petersen grab, 49 m, 2 specimens (USNM 187870); Sta. 745, 64'46.23'S, 64 04.58'W, 31 Dec 1971, 20 m, 1 specimen (USNM 187871); Sta. 746, 64 $46.32^{\prime}$ 'S, $64^{\circ} 04.45^{\prime} \mathrm{W}, 31 \mathrm{Dec} 1971,26$ m, 3 specimens (USNM 187872); Sta. 801, 62 $43^{\prime}$ 'S, $61^{\circ} 02.5^{\prime} \mathrm{W}, 14$ Jan 1972, 73 m, 20 specimens (USNM 187873); Sta. 1018, 64²42'S, 62³8'W, 15 Dec 1971, 97 m, 2 specimens (USNM 187874); Sta. 1058, 62 $2^{\circ} 19.0^{\prime} \mathrm{S}, 59^{\circ} 11.4^{\prime} \mathrm{W}, 19 \mathrm{Dec} 1971,44 \mathrm{~m}, 1$ specimen (USNM 187875); Sta. 1060, 62¹9.0’S, 59¹1.4'W, 19 Dec 1971, $44 \mathrm{~m}, 10$ specimens (USNM 187876); Sta. 1063, 62 19.0'S, $59^{\circ} 11.4^{\prime} \mathrm{W}, 19 \mathrm{Dec} 1971,4$ specimens (USNM 187878), 1 speci-

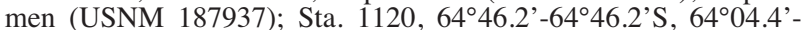
640.3'W, 7 Mar 1972, 25-31 m, 16 specimens (USNM 187879); Sta. $1121,64^{\circ} 46.8^{\prime}-64^{\circ} 46.4^{\prime} \mathrm{S}, 64^{\circ} 05.3^{\prime}-64^{\circ} 04.6^{\prime} \mathrm{W}, 7$ Mar 1972 , 38-70 m, 5 specimens (USNM 187880); Sta. 1131, 6446.4'- 
6446.3'S, 64 ${ }^{\circ} 046^{\prime}-64^{\circ} 045^{\prime} \mathrm{W}, 10 \mathrm{Mar} 1972,22-45 \mathrm{~m}, 3$ specimens (dried out) (USNM 187881); Sta. $1135,65^{\circ} 02.4^{\prime}-65^{\circ} 02.4^{\prime} \mathrm{S}$, 63⒌4'-63 ${ }^{\circ} 55.6^{\prime} \mathrm{W}, 13$ Mar 1972, 145-297 m, 1 specimen (USNM

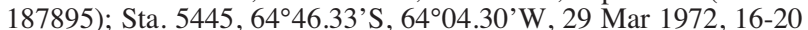
$\mathrm{m}, 56$ specimens (USNM 187882); Cruise 731, Sta. 1786, $64^{\circ} 46.25^{\circ} \mathrm{S}, 64^{\circ} 04.85^{\prime} \mathrm{W}, 18$ Feb 1973, 24 M, 61 specimens

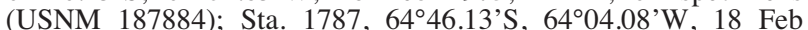
1973,15 m, 4 specimens (USNM 187885); Sta. 1790, 64 $46.43^{\prime} \mathrm{S}$, $64^{\circ} 04.8^{\prime} \mathrm{W}, 18$ Feb $1973,35 \mathrm{~m}, 4$ specimens (USNM 187886); Sta. 1791, 64 $46.43^{\prime} \mathrm{S}, 6^{\circ} 04.87^{\prime} \mathrm{W}, 18$ Feb 1973, $67 \mathrm{~m}, 2$ specimens (USNM 187887); Sta. 1792, 64 $46.6^{\prime} \mathrm{S}, 64^{\circ} 05^{\prime} \mathrm{W}, 18 \mathrm{Feb} 1973,70$ m, 3 specimens (USNM 187888); Sta. $1804,64^{\circ} 46.43^{\prime}-64^{\circ} 46.25^{\prime} \mathrm{S}$, $64^{\circ} 04.28^{\prime}-64^{\circ} 04.85^{\prime} \mathrm{W}, 18$ Feb $1973,16-24 \mathrm{~m}, 5$ specimens (USNM 187889); Sta. 1897, 64²46.68' S, 6404.07'W, 06 Mar 1973, 22 m, 3 specimens (USNM 187890); Sta. 1902, 6446.92'S, 640.42'W, 6 Mar 1973, 49-61 m, 5 specimens (USNM 187891); Sta. $1904,64^{\circ} 47.02^{\prime} \mathrm{S}, 6^{\circ} 04.55^{\prime} \mathrm{W}, 06$ Mar 1973, 26 m, 1 speci-

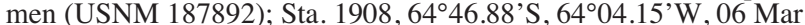
1973, 58 m, 6 specimens (USNM 187893); Sta.1912, 6446.88'$64^{\circ} 46.87^{\prime} \mathrm{S}, 64^{\circ} 03.58^{\prime}-64^{\circ} 03.15^{\prime} \mathrm{W}, 6$ Mar $1973,25-55 \mathrm{~m}, 14 \mathrm{spec}-$ imens (USNM 187894). Cruise 824, Sta. 3-2, 65¹4.00'S, 64 15.50’W, 15 Mar 1982, 60 m, 15 specimens (USNM 187933); Sta. 3-3, 65'14.00'S, 64 $15.50^{\prime} \mathrm{W}, 15$ Mar $1982,59 \mathrm{~m}, 4$ specimens (USNM 187925); Sta. 3-4, 65¹4.00’S, 64¹5.50’W, 15 Mar 1982 , $60 \mathrm{~m}, 11$ specimens (USNM 187938); Sta. 3-7, 65¹4.00'S, $64^{\circ} 15.50^{\prime} \mathrm{W}, 15$ Mar 1982, 56 m, 30 specimens (USNM 187935), 1

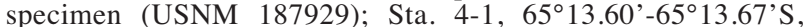
$64^{\circ} 14.72^{\prime}-64^{\circ} 15.07^{\prime} \mathrm{W}, 16$ Mar $1982,49-58 \mathrm{~m}, 11$ specimens (USNM 187918); Sta. 16-2, 64 $19.5^{\prime}$ 'S, 62 ${ }^{\circ} 59.58^{\prime} \mathrm{W}, 22$ Mar 1982 , $85 \mathrm{~m}, 4$ specimens (USNM 187926); Sta. 16-3, 64 $19.5^{\prime} \mathrm{S}$, 62 $2^{\circ} 9.58^{\prime} \mathrm{W}, 22$ Mar 1982, 85 m, 5 specimens (USNM 187931); Sta. 16-4, 64 $4^{\circ} 19.50^{\prime} \mathrm{S}, 62^{\circ} 59.58^{\prime} \mathrm{W}, 22$ Mar $1982,87 \mathrm{~m}, 5$ specimens (USNM 187928); Sta. 17-1, 64 $19.5^{\prime} \mathrm{S}, 62^{\circ} 59.58^{\prime} \mathrm{W}, 22 \mathrm{Mar}$ $1982,55-80$ m, 3 specimens (USNM 187915); Sta. 22-1, 64²2.30'$64^{\circ} 22.10^{\prime} \mathrm{S}, 61^{\circ} 28.3^{\prime}-61^{\circ} 29.30^{\prime} \mathrm{S}, 23 \mathrm{Mar} 1982,60-152 \mathrm{~m}, 1$ specimen (USNM 187920); Sta. 24-1, 64 $15.20^{\prime}-64^{\circ} 14.5^{\prime} \mathrm{S}, 61^{\circ} 27.5^{\prime}$ 61 25.90'W, 23 Mar 1982, 540-605 m, 1 specimen (USNM 187921); Sta. $28-1,64^{\circ} 14.20^{\prime}-64^{\circ} 13.80^{\prime} \mathrm{S}, 62^{\circ} 35.10^{\prime}-62^{\circ} 34.10^{\prime} \mathrm{W}$, 24 Mar 1982, 70-98 m, 9 specimens (USNM 187924); Sta. 30-1, $64^{\circ} 14.10^{\prime} \mathrm{S}-64^{\circ} 14.20^{\prime} \mathrm{S}, 62^{\circ} 32.30^{\prime}-62^{\circ} 34.90^{\prime} \mathrm{W}, 25 \mathrm{Mar} 1982,90-$ $135 \mathrm{~m}, 4$ specimens (USNM 187927); Sta. 32-1, 64³7.00'6438.00’S, 62 $2^{\circ} 50.80^{\prime}-62^{\circ} 51.60^{\prime} \mathrm{W}, 25$ Mar 1982, 640-670 m, 16

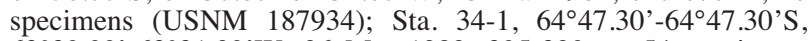

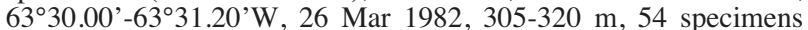
(USNM 187916), 1 specimen (USNM 187922); Sta. 34-2,

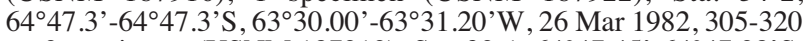

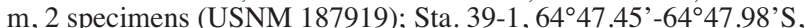

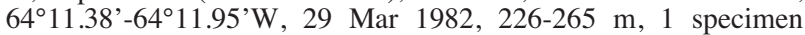
(USNM 187932); Sta. 41-1, 6448.42'S, 6407.00'W, 29 Mar 1982, Blake trawl, 88-112 m, 10 specimens (USNM 187914); R/V Staten Island, coll. Schmitt, 434ㅇ' S, $172^{\circ} 43^{\prime} \mathrm{E}, 11$ Dec 1962, depth not recorded, 1 specimen (USNM 187900).

Description. Colour in alcohol white to brown; length up to $13 \mathrm{~mm}$ for 23-28 chaetigers. Prostomium conical, gently rounded at tip, no eyes present; two well-developed nuchal organs at base of prostomium. Eversible proboscis a soft pouch. Branchiae from chaetiger 2, absent from last 4-5 chaetigers; branchiae slender, cirriform, pointed at tip, usually arched towards/over dorsal surface. Ventral and lateral grooves extending along length of body. Chaetae similar throughout, broad, plain capillaries. Parapodia low, rounded. Segments uniannulate. Anal tube cylindrical, terminating in a circlet of small subequal papillae, with one longer papilla on ventral (inferior) side; anal tube easily detached, often missing.
Methyl Green staining pattern. No particular pattern, although the ventral groove stains darker than surrounding tissue and appears as a long thin ventral stripe. The lateral area along the parapodia also stains a bit darker. Otherwise, the stain is taken up uniformly.

Remarks. The name Ammotrypane aulopyge was used by Ehlers in 1897 to describe a new species; he later (1901) determined that the name was a homonym of a Grube species, and therefore replaced it with $A$. syringopyge.

This species was the most common opheliid in the collections. The anal funnel is easily detached and care should be taken that specimens lacking the funnel are not misidentified as another species. One specimen in the collection has a single gill with three branches; this anomaly is not considered significant at this time. Several specimens (e.g. from Hero 1912 and several other samples) were full of large eggs.

Distribution. Strait of Magellan; South Georgia; Alexander Island; Kerguelen Islands: 9-876 m; off Argentine coast $\left(34^{\circ} \mathrm{S}\right) 83-199 \mathrm{~m}$.

\section{Genus Travisia Johnston, 1840}

Travisia Johnston, 1840. Type species: Travisia forbesii Johnston, 1840

Dindymenides (Kinberg, 1866) Type species: Dindymene concinna (= Dindymenides concinna) by monotypy. (Homonym of crustacean, renamed by Chamberlin 1919). Fide Dauvin and Bellan, 1994.

Kesun Chamberlin, 1919. Type species: Kesun fusus Chamberlin, 1919. Fide Dauvin and Bellan, 1994.

Diagnosis. Body stout, pointed at both ends, fusiform or grub-like, rounded cylindrically, without lateral or ventral grooves, or grooves very reduced. Prostomium small, smooth, rounded, conical or truncate, without eyes or processes; nuchal organs present. First chaetiger anterior to mouth. Parapodia reduced, small and smooth, or entirely absent. Branchiae present or absent; branchiae cirriform or branched, may be annulated; branchiae when present from chaetiger 2 or 3 . Interramal lateral sensory organs present, lateral eyes absent. Chaetae simple capillaries, may be hispid. Pygidium small, cylindrical, longitudinally furrowed, with ring of stout unequal papillae or without lobes or papillae. Segments annulated, with posterior segments telescoped or forming folds ending in dorsal lappets 
dorsal and ventral to parapodial rami, these parapodial lappets low and rounded, large and leaflike, or pointed and triangular.

Remarks. Chamberlin (1919) renamed the genus Dindymene, established by Kinberg (1866), to Dindymenides, having determined that the former was preoccupied in the Crustacea. Dindymenides has not been widely reported (perhaps three records) and has consisted of a single species, $D$. concinna, described by Kinberg from 5-20 m in Algoa Bay, in tropical South Africa. Hartman (1948) examined Kinberg's material and was of the opinion that the type specimen had been unnaturally flattened in the vial, and that, contrary to Kinberg's description, the first chaetae were in front of the oral aperture, rather than behind it, thus questioning the validity of the genus. Bellan et al. (1990), following Fauchald (1977), retained the genus as distinct, but Dauvin and Bellan (1994) preferred to consider it invalid, citing Hartman (1948) and a recent opinion by Fauchald (in litt.). Chamberlin (1919) also established a new genus, Kesun, which he differentiated from the related genus Travisia by the complete absence of branchiae.

Following a cladistic analysis of morphological characters, Dauvin and Bellan (1994) synonymized both Kesun and Dindymenides with Travisia, and recognized a total of 27 species and three subspecies in the genus. Characters that are important at the species level include the presence of lobes or papillae on the pygidium, the position and relative size of the nephridiopores, and the total number of chaetigers, which appears to be relatively stable in most, but not all, species (Dauvin and Bellan, 1994). Blake (2000) also found the number of segments to be important in Travisia; however, if the number of segments is not fixed for the parent species, the validity of a (sub)species based solely on a differing number of segments is highly questionable. For example, Travisia kerguelensis is described as having 23-27 segments, and the subspecies T. kerguelensis gravieri as having 25. Similarly, T. olens has 29-36 segments, and the subspecies T. olens novae zealandiae, 38-40. Other characters are also necessary to support the retention of the species or subspecies. In the example of T. kerguelensis gravieri, the original illustrations and description by Monro suggest that this form, in lacking pygidial papillae but having two short dorsal anal cirri, may be separate from T. kerguelensis at the species, rather than the subspecies, level.
Hartman (1966, 1967, 1978) recorded seven species of Travisia from Antarctica and surrounding waters, including T. antarctica Hartman 1967; T. kerguelensis McIntosh, 1885; T. kerguelensis gravieri Monro, 1930; T. lithophila Kinberg, 1866; T. nigrocincta Ehlers, 1913; T. olens Ehlers, 1897; and T. profundi Chamberlin, 1919. She also recorded Kesun abyssorum Monro, 1930, which Dauvin and Bellan (1994) referred to Travisia glandulosa McIntosh, 1879. Of these eight species, three are in the present collections, plus one new species from the Antarctic Peninsula; the other new Travisia is from additional collections off Peru.

\section{Travisia antarctica Hartman, 1967}

(emended 1978)

Travisia antarctica Hartman, 1967, p. 139. Hartman, 1978, p. 184185 , figs. 31a-c.

Material examined. R/V Eltanin Cruise 3, Sta. $78,31^{\circ} 25^{\prime}-31^{\circ} 27^{\prime} \mathrm{S}$, $72^{\circ} 35^{\prime}-72^{\circ} 35^{\prime} \mathrm{W}, 26$ Jun $1962,5609-6010 \mathrm{~m}, 4$ specimens (USNM 187950); Cruise 7, Sta. 457, no depth or position recorded, sampled by engine strainer, 2 specimens (USNM 187961); Sta. 475, 57 11 '57 $14^{\circ} \mathrm{S}, 4^{\circ} 53^{\prime}-44^{\circ} 47^{\prime} \mathrm{W}, 14$ Feb 1963, $3382 \mathrm{~m}, 1$ specimen

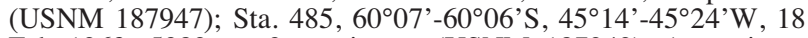
Feb $1963,5289 \mathrm{~m}, 2$ specimens (USNM 187949), 1 specimen

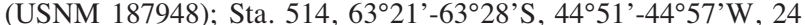
Feb 1963, Blake trawl, 3587-3660 m, 1 specimen (USNM 187945); Sta. 519, 66 $66^{\circ}$ ' $-66^{\circ} 29^{\prime} \mathrm{S}, 48^{\circ} 11^{\prime}-48^{\circ} 10^{\prime} \mathrm{W}, 28$ Feb 1963, $3722 \mathrm{~m}$, 1 specimen (USNM 187954); Cruise 8, Sta. 599, $58^{\circ} 11^{\prime} \mathrm{S}-58^{\circ} 12^{\prime}$ 'S, $25^{\circ} 48^{\prime}-25^{\circ} 45^{\prime} \mathrm{W}$, Menzies trawl, 03 May 1963, 2496-2527 m, 1 specimen (USNM 187946); Sta. 656, 59³3'-59³4'S, 27¹2'$27^{\circ} 11^{\prime} \mathrm{W}, 29$ May 1963, 1171-1226 m, 1 specimen (USNM 187951); Cruise 9, Sta. 691, 56 $25^{\circ}-56^{\circ} 29^{\prime} \mathrm{S}, 36^{\circ} 57^{\prime}-37^{\circ} 05^{\prime} \mathrm{W}, 27$ Aug 1963, 3118 m, 2 specimens (USNM 187952); Sta. 693, 56 53'$56^{\circ} 54^{\prime} \mathrm{S}, 37^{\circ} 31^{\prime}-37^{\circ} 32^{\prime} \mathrm{W}, 28$ August 1963, Menzies trawl, 3138 m, 1 specimen (USNM 187905), 1 specimen (USNM 187905); Sta. $711,58^{\circ} 43^{\prime} \mathrm{S}, 33^{\circ} 30^{\prime}-33^{\circ} 23^{\prime} \mathrm{W}, 04$ Sep 1963, Menzies trawl, 2983$3331 \mathrm{~m}, 2$ specimens (USNM 187906), 1 specimen (USNM 187906); Sta. $724,54^{\circ} 05^{\prime}-54^{\circ} 04^{\prime} \mathrm{S}, 33^{\circ} 44.3^{\prime}-33^{\circ} 37^{\prime} \mathrm{W}, 09$ Sep $1963,2714-2727$ m, 1 specimen (USNM 187955); Sta. $735,5^{\circ} 02^{\prime}$ $53^{\circ} 02^{\prime} \mathrm{S}, 37^{\circ} 02^{\prime}-37^{\circ} 38^{\prime} \mathrm{W}, 13$ Sep $1963,3056-3102 \mathrm{~m}, 1$ specimen

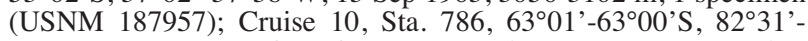
$82^{\circ} 22^{\prime} \mathrm{W}, 24$ Oct $1963,4602 \mathrm{~m}, 2$ specimens (USNM 187959); Cruise 16, Sta. $1403,41^{\circ} 42^{\prime}-41^{\circ} 42^{\prime}$ S, $175^{\circ} 29^{\prime}-175^{\circ} 22^{\prime} \mathrm{E}, 31$ Jan 1965, 946-951 m, 1 specimen (USNM 187956); Cruise 17, Sta. 2-

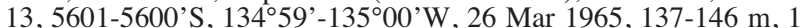
specimen (USNM 187958); Cruise 22, Sta. $1578,55^{\circ} 49^{\prime}-55^{\circ} 56^{\prime} \mathrm{S}$, $22^{\circ} 11^{\prime}-22^{\circ} 09^{\prime} \mathrm{W}, 04$ Mar 1966, 4236-4273 m, 1 specimen (USNM 187953); Sta. $1585,56^{\circ} 11^{\prime}-56^{\circ} 19^{\prime} \mathrm{S}, 38^{\circ} 36^{\prime}-38^{\circ} 32^{\prime} \mathrm{W}, 09 \mathrm{Mar}$ 1966, 2869-3038 m, 1 specimen (USNM 187960); Cruise 32, Sta. $2076,7^{\circ} 25^{\prime} \mathrm{S}, 170^{\circ} 34^{\prime} \mathrm{W}, 30$ Jan 1968 , camera grab, $558 \mathrm{~m}, 1$ specimen (USNM 187943); Cruise 38, Sta. 8, 61 ${ }^{\circ} 55.64$ 'S, 14904.47'E, 09 Apr 1969, Blake/Menzies, 3756 m, 1 specimen (USNM 187944); Sta. 11, gr 31, 4945.57'S, $152^{\circ} 36.18^{\prime} \mathrm{E}, 4304 \mathrm{~m}$, 30 Apr 1969, 1 specimen (USNM 187942). R/V Glacier, Cruise 1, Sta. 1, 7406'S, 39³8'W, 08 Feb 1968, 731 m, 1 specimen (USNM $187940) ; \mathrm{R} / \mathrm{V}$ Islas Orcada Cruise 575, Sta. 51, 57⒉6'S, 26³4.0'W, 26 May 1975, 2248-2402 m, 2 specimens (USNM 187941).

Description. Length $35 \mathrm{~mm}$, width $11 \mathrm{~mm}, 28$ segments, last four segments achaetous, followed by anal cylinder. Body surface granular, most conspicuous in anterior third. Prostomium smooth, broadly 
triangular depressed lobe. Peristomium smooth, trapezoidal, ventrally appearing to be broad, smooth with mid-longitudinal fissure. Mouth a transverse slit between chaetigers 1 and 2. Chaetiger 1 biramous, with well-developed and well-separated chaetal fascicles, slightly larger than fascicles on chaetiger 2. Branchiae first present, but small, from chaetiger 2, number 20-21 pairs and are largest in anterior segments. Branchial segments followed by three abranchiate chaetigerous segments, and these by four abranchiate, achaetigerous segments.

Each segment with transverse circlets of beadshaped papillae arranged in several rows. Parapodia located on ring with largest papillae. The first 16 or 17 segments triannulate, next six segments biannulate, last five uniannulate. Interramal sense organs present. Nephridiopores located a short distance anteroventrally to neuropodia. Chaetae all long, slender capillaries with smooth edges.

Anal cylinder slender, surrounded by circle of short papillae.

Methyl Green staining pattern. No pattern, although the stain outlines the papillae, making the size and arrangement easier to observe.

Remarks. The species was described as being unique because it has 28 segments, of which 20 or 21 are branchial; the last four segments lack chaetae. Many of the specimens examined have lost branchiae. The most notable feature of this species is the rows of papillae on the body surface, which, as Hartman wrote, resemble closely-strung beads.

The majority of records for this species are from $2000 \mathrm{~m}$ and deeper; however, good specimens from approximately $140 \mathrm{~m}$ were recorded from the present material.

Distribution. Antarctic waters, 137-6010 m.

\section{Travisia kerguelensis McIntosh, 1885}

Travisia kerguelensis McIntosh, 1885, pp. 357B359, pl. 43, fig. 10, pl. 36A, figs. 1,2. Ehlers, 1897, p. 97. Ehlers, 1900, p. 13 . Ehlers, 1901, p. 170. Willey, 1902 p. 276. Ehlers, 1912, p. 23. Monro, 1930, pp. 165-167, fig. 67. Augener, 1932, pp. 43-44. Monro, 1936, p. 165. Monro, 1939, p. 133. Fauvel, 1941, pp. 290-291.CHartman, 1953, p. 48. Knox, 1962, p. 346. Hartman, 1966, p. 54, pl. XVII, figs. 4-5. Hartman, 1978, p. 185.

Material examined. R/V Eltanin Cruise 6, Sta. 344, 540.'', $58^{\circ} 48^{\prime} \mathrm{W}-58^{\circ} 45^{\prime} \mathrm{W}, 04 \mathrm{Dec} 1962$, Menzies trawl, ca. $119 \mathrm{~m}, 1 \mathrm{spec}-$ imen (USNM 187964); Sta. $363,57^{\circ} 09^{\prime} \mathrm{S}-58^{\circ} 00^{\prime} \mathrm{S}, 58^{\circ} 58^{\prime} \mathrm{W}-$ $58^{\circ} 50^{\prime} \mathrm{W}, 07$ Dec 1962, depth not recorded, Phleger corer, 1 specimen (USNM 187974); Sta. 369, 54 $04^{\prime}-56^{\circ} 01^{\prime} \mathrm{S}-63^{\circ} 35^{\prime}-63$ 44'W, rock dredge, 12 Dec 1962, 247-293 m, 1 specimen (USNM 1078715); Cruise 11, Sta. $960,52^{\circ} 40^{\prime} \mathrm{S}-52^{\circ} 36^{\prime} \mathrm{S}$, $74^{\circ} 58^{\prime} \mathrm{W}$ $74^{\circ} 55^{\prime} \mathrm{W}, 06$ Feb 1964, Blake trawl, $64 \mathrm{~m}, 1$ specimen (USNM 187969); Cruise 21, Sta. 288, 06 Jan 1966, 52 $2^{\circ} 52^{\prime}$ S-52 ${ }^{\circ} 56^{\prime} \mathrm{S}$, $75^{\circ} 18^{\prime}-75^{\circ} 23^{\prime} \mathrm{W}$, Blake trawl, 119-329 m, 1 specimen (USNM 187966); Cruise 25 , Sta. $344,49^{\circ} 43^{\prime} \mathrm{S}-49^{\circ} 39^{\prime} \mathrm{S}, 110^{\circ} 16^{\prime} \mathrm{W}$ $110^{\circ} 21^{\prime} \mathrm{W}, 20$ Oct 1966, $1030 \mathrm{~m}, 1$ specimen (USNM 187967). R/V Glacier, Cruise 2, Sta. 1, 74²28.1'S, 30³1.7'W, 24 Feb 1968, $513 \mathrm{~m}, 5$ specimens (USNM 187971); R/V Hero Cruise 702, Sta.

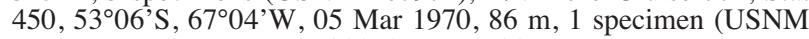

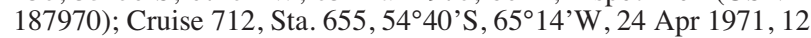
m, 2 specimens (USNM 187973); Cruise 715, Sta. 883, 54²47.2'S $64^{\circ} 05^{\circ} \mathrm{W}, 29$ Oct 1971 , grab, $31 \mathrm{~m}, 1$ specimen (USNM 187972); Cruise 721, Sta. 776, 08 Jan 1972, 62 56.1'S-62 $2^{\circ} 56.7^{\circ} \mathrm{S}$, $60^{\circ} 49.0^{\prime} \mathrm{W}-60^{\circ} 49.6^{\prime} \mathrm{W}$, Blake trawl, $109 \mathrm{~m}, 1$ specimen (USNM 187965); Sta. 795 , 12 Jan $1972,62^{\circ} 58.8^{\prime}$ S, $60^{\circ} 47.3^{\prime} \mathrm{W}$, Petersen grab, $55 \mathrm{~m}, 1$ specimen (USNM 187968); Sta. 796, 12 Jan 1972, $62^{\circ} 58.6^{\prime} \mathrm{S}, 60^{\circ} 48.7^{\prime} \mathrm{W}, 65 \mathrm{~m}, 2$ excellent specimens (USNM 187963).

Description. Length 20 to $40 \mathrm{~mm}$, width 7 to 8 $\mathrm{mm}$, segments number 23 to 27 . Body whitish, appearing minutely dotted (papillated), thickest at middle and tapering to both ends. First chaetiger with chaetal fascicles shifted dorsally; chaetal fascicles well separated, with interramal sensory pits visible from chaetiger 1 to about chaetiger 16. Anterior chaetigers without obvious parapodia; last 9-11 segments with parapodia and lateral crenelations, the degree of indentation increasing posteriorly. Branchiae present from chaetiger 2, continuing almost through to posterior end; may be retractile, inconspicuous. Anal cylinder abrupt after last parapodial segment, slender anal tube about as long as last four segments.

\section{Methyl Green staining pattern. No pattern.}

Remarks. The notched shape of the posterior parapodia is distinctive. Earlier descriptions have used the words "lacinated" or "crenulated" to describe this shape, but those words imply a structure that has a fringed margin (lacinated) or has low, rounded teeth (crenulated), and do not convey the actual shape of the body part being described. "Crenellated," meaning "indented or notched," is more accurate.

Distribution. Kerguelen Islands, Southern Indian, Southern Atlantic, Antarctic Oceans, in 201030 m. (0-1837 m re: Dauvin and Bellan, 1994)

\section{Travisia monroi new name}

Travisia kerguelensis gravieri Monro, 1930, p. 167, fig. 68. Hartman, 1966, p. 54, pl. XVII, fig. 6.

Material examined. R/V Eltanin, Cruise 6, Sta. 410, 61 ${ }^{\circ} 18^{\prime}-$ $61^{\circ} 20^{\prime} \mathrm{S}, 56^{\circ} 09^{\prime}-56^{\circ} 10^{\prime} \mathrm{W}, 31$ Dec 1962, 220-240 m, 1 specimen (USNM 187962). 
Etymology. This species is named after Dr. C.C.A. Monro, who originally described this species.

Remarks. The subspecies T. kerguelensis gravieri was described by Monro (1930) from 259-315 m off the Palmer Archipelago. Although Dauvin and Bellan (1994) recognized the subspecies, they pointed out that separation of the closely related forms is difficult, since the number of segments overlap. However, the differences noted by Monro (1930, p.167) focus on the absence of papillation (or laciniation) of the terminal segments, and in the presence of a pair of short dorsal anal cirri at the end of the anal cylinder. The single specimen in the present material has the two longer dorsal anal cirri described by Monro, and although the parapodia are developed in several posterior chaetigers as they are in T. kerguelensis, there is no hint of crenellations on any of the posterior segments. The specimen has numerous white sand grains adhering to the body surface, but these are not papillae, and indeed papillae seem to be absent from the posterior chaetigers.

These differences suggest that this form is a valid full species rather than subspecies, and herein is raised to species level. However, the name Travisia kerguelensis gravieri is a homonym of a species described by McIntosh (1908), and a new name is therefore required for the Monro species.

Distribution. Antarctic Peninsula, 220-315 m

Travisia palmeri $\mathrm{n} . \mathrm{sp}$. (Fig. 2)

Material examined. Antarctic Peninsula, R/V Hero, Cruise 824, Sta.

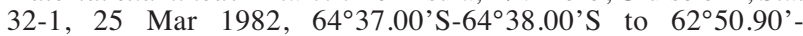
$62^{\circ} 51.60$ 'W, 640-670 m, 1 specimen (Holotype, USNM 1078731).

Description. Holotype a large, bulky specimen, measuring $22 \mathrm{~mm}$ long and from $2 \mathrm{~mm}$ (anterior tip) to $5 \mathrm{~mm}$ (posterior) wide for 24 chaetigers (Fig. 2A). Epidermis highly papillated, papillae all small, similar; segments triannulate except for last four or five. Parapodial lappets present from chaetigers 15-16, becoming larger through last segment. Notopodial and neuropodial chaetal rami well separated; chaetae all capillaries. Branchiae from chaetiger 2, triangular, elongated, annulated (Fig. 2B). Branchiae number 22 pairs, pairs $1-3$ and 5-8 short, pair 4 with long tip, pairs 9-12 very long, extending the length of 3-4 chaetigers, distal portion becoming very long, thin; pairs 13-14 shorter, but still with

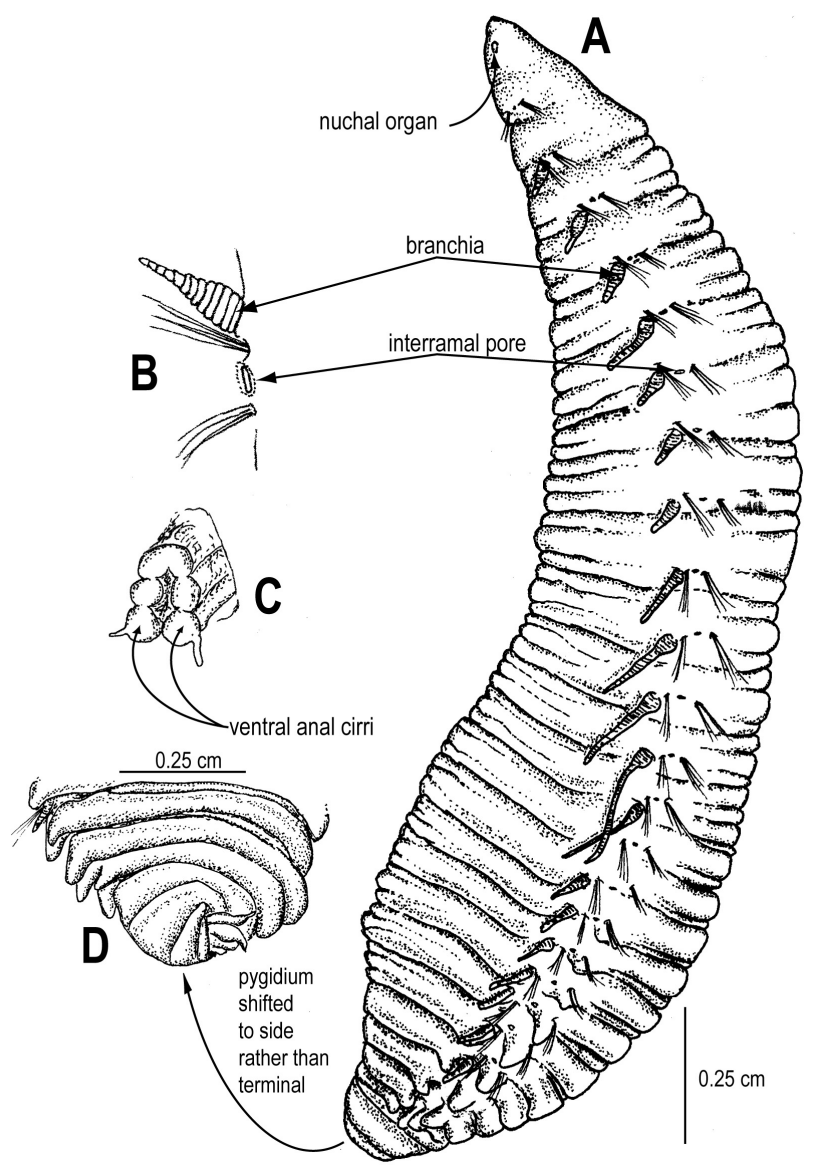

FIG . 2. - Travisia palmeri n. sp. (Holotype, R/V Hero, Cruise 824, Sta. 32-1, 25 Mar 1982, USNM 1078731). A. Entire animal in lateral view; B, median parapodium; C. pygidium, posterior view; D, pygidium, dorso-posterior view.

long tip; thereafter branchiae very short. Lateral pores obvious between parapodial rami on chaetigers 1-20 (Fig. 2B), either hidden or absent on last four chaetigers. Pygidium shifted laterally, appearing to terminate on side of animal (Fig. 2D); anal aperture appearing puckered, with two larger, broad ventral cirri (Fig. 2C).

Methyl Green staining pattern. No particular staining pattern, although when first removed from the stain, the tip of the prostomium, lips of the lateral pores, and internal rim of the anal opening did not retain any stain. As the stain differentiates, the branchiae clear first, then segmental bands (between the annulations on the segments), and eventually all of the body tissues are clear of stain.

Etymology. This species is named after the general location of the first collection of this species, near Palmer Station on the Antarctic Peninsula. 


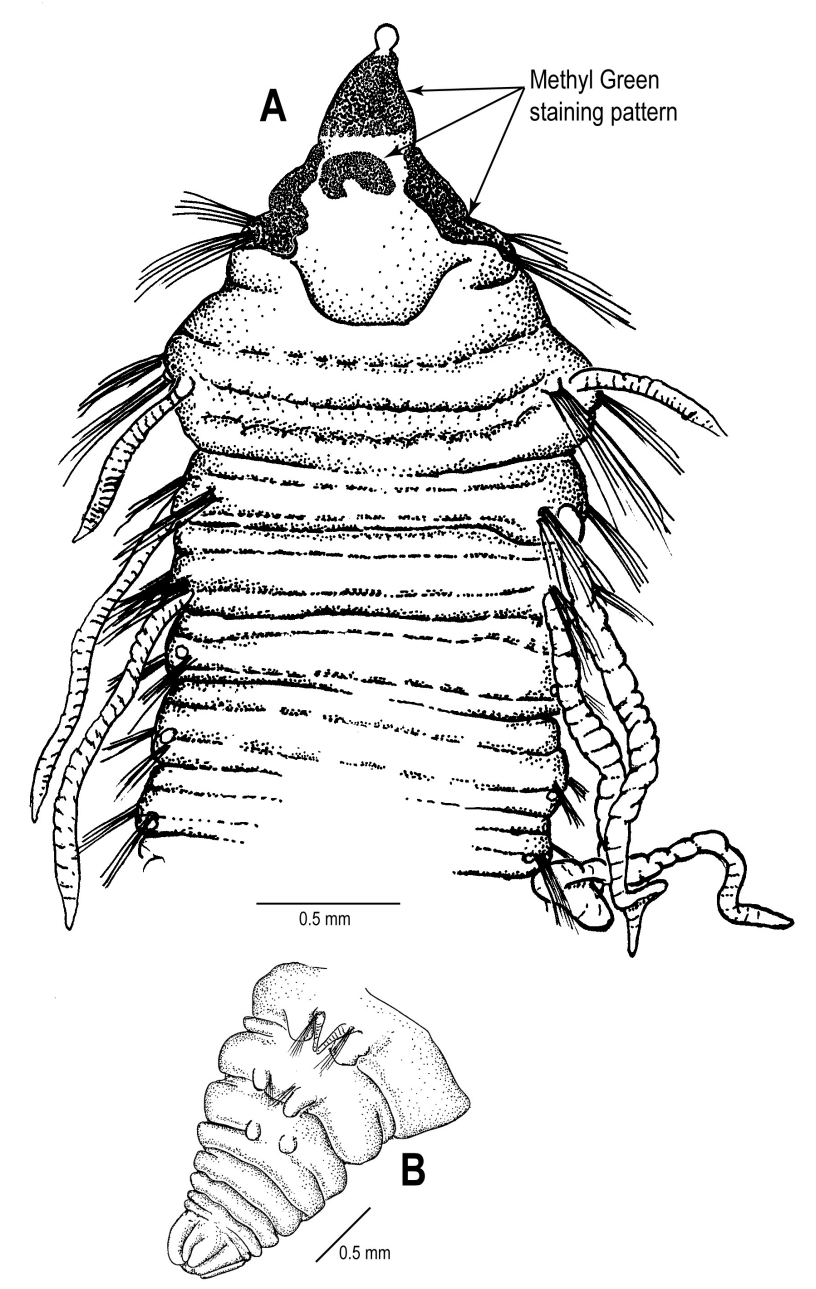

FIG . 3. - Travisia tincta n. sp. (Holotype, R/V Anton Brun, Cruise 17, Sta. 660-G, 26 June 1966, USNM 1078732). A, Anterior end, dorsal view; B, pygidium, lateral view.

Remarks. The long gills on chaetigers 9 through to 12 may be similar to the long branchiae in T. lithophil$i a$; however, that species has 52-53 chaetigers compared with the 22 chaetigers ( 26 segments) in the new species. The very odd orientation of the pygidium is not understood at this time. Either it is due to an artifact that occurred during collection and fixation (NJM), or it is real (JAB). If the latter, it is very unusual.

Distribution. Antarctic Peninsula, ca. 650 m.

Travisia tincta $\mathrm{n} . \mathrm{sp}$.

(Fig. 3)

Material examined. Off Lima, Peru, R/V Anton Brun, Cruise 17, Sta. 660-G, $12^{\circ} 58^{\prime} \mathrm{S}, 77^{\circ} 16^{\prime} \mathrm{W}, 26$ June $1966,1000 \mathrm{~m}, 1$ specimen (HOLOTYPE, USNM 1078732).

Description. Holotype complete, damaged in mid-body region; measures $13 \mathrm{~mm}$ long and $2 \mathrm{~mm}$ wide for 21 chaetigers plus pygidium. Proboscis soft pouch, everted on holotype. Segments biannulate in anterior, triannulate posteriorly. No parapodial lamellae on chaetiger 1, both notochaetae and neurochaetae present (Fig. 3A); subsequent parapodia with very small lamellae.

Branchiae from chaetiger 2, annulated (have appearance of vacuum cleaner hose); branchiae very long on chaetigers $2-12$, short on next eight chaetigers, absent from last chaetiger, each branchia arising just posterior to notochaetae: branchial formula $-1 \mathrm{~A}+11$ long +8 short $+1 \mathrm{~A}$. Pygidium with five annulations (Fig. 3B). Anal aperture surrounded by ring of indentations or furrows (Fig. 3B).

Methyl Green staining pattern. A very distinctive staining pattern, with the majority of the prostomium and peristomium staining deeply, except for the tip and a bright white (non-staining) band near the middle of the prostomium (Fig. 3A).

Etymology. The species name is taken from the Latin tinctus, and refers to the retention of Methyl Green stain by this species.

Remarks. The extremely long branchiae in the middle body segments, plus the anterior Methyl Green staining pattern are unique.

Distribution. off Lima, Peru; 1000 m.

\section{ACKNOWLEDGEMENTS}

This study was funded by a grant from the Smithsonian Institution, Washington, D.C., to James A. Blake. Additional support was provided by the National Science Foundation under Grant No. DEB0118693 (PEET) to James A. Blake, University of Massachusetts, Boston. We thank Dr. Kristian Fauchald, Smithsonian Institution, for assistance in making this study possible, and for translating a species description from Norwegian. We also thank two anonymous reviewers for their comments.

\section{REFERENCES}

Augener, H. - 1932. Antarktische und Antiboreale polychaeten nebst einer Hirudinae. Scientific Results of the Norwegian Antarctic Expedition 1927-28. Norske Videnskaps Akad. Oslo. 86 pp.

Bellan, G. and J.C. Dauvin. - 1991. Phenetic and biogeographic relationship in Ophelia (Polychaeta, Opheliidea). Bull. Mar. 
Sci. 48: 544-558

Bellan, G., D. Bellan-Santini, and J.C. Dauvin. - 1990. Phénétique et phylogénie des Opheliidae (Annélides Polychètes). C.R Acad. Sci. Paris, 310, sér. III: 175-181.

Blake, J.A. - 2000. Chapter 7. Family Opheliidae Malmgren, 1867. In: J.A. Blake, B. Hilbig, and P.H. Scott (eds.), Taxonomic Atlas of the Santa Maria Basin and Western Santa Barbara Channel. Volume 7. The Annelida Part 4. Polychaeta: Flabelligeridae to Sternaspidae, pp. 145-168. Santa Barbara Museum of Natural History.

Chamberlin, R.V. - 1919. The Annelida Polychaeta. Mem. Mus Comp. Zool. Harvard, 48: 1-514.

Dauvin, J.C. and G. Bellan. - 1994. Systematics, ecology and biogeographical relationships in the sub-family Travisiinae (Polychaeta, Opheliidae). In: J.C. Dauvin, L. Laubier and D.J. Reish (eds.), Actes de la 4ème Conférence internationale des Polychètes. Mém. Mus. natn. Hist. nat. 162: 169-184.

Ehlers, E. - 1897. Polychaeten. In: Hamburger Magalhaenischen Sammelreise. 148 pp., 9 pls. Friedrichsen \& Co., Hamburg.

Ehlers, E.- 1900a. Magellanische Anneliden gesammelt während der schwedishchen Expedition nach den Magellansländern. $K$. Ges. Wiss. Göttingen, Nachr., Math-Phys. K1.pp. 1-18.

Ehlers, E.- 1900b. Magellanische Anneliden gesammelt während der schwedishchen Expedition nach den Magellansländern. $K$. Ges. Wiss. Göttingen, Nachr., pp. 206-223.

Ehlers, E. - 1901. Die Polychaeten des magellanischen und chilenischen Strandes. Ein faunistischer Versuch. Festschrift zur Feier des Hundertfunfzigjahrigen Bestehens der koniglichen Geselschaft der Wissenschaften zu Gottingen. pp. 1-232, pls. 1-25

Ehlers, E. - 1912. Polychaeten. Hamburger Magellhaenische Sammelreise, Hamburg.

Ehlers, E. - 1913. Die Polychaeten-Sammlungen der deutschen Sudpolar Expedition 1901-1903. Deutsche Sudpolar-Exped., 13(4): 397-598.

Elias, R. and C.S. Bremec. - 2003. Opheliidae (Polychaeta) of the southwestern Atlantic coast: first record of the genus Armandia in Argentine waters, with the description of Armandia loboi sp. n. Bull. mar. Sci. 72(1): 181-186.

Elias, R., C.S. Bremec, P.C. Lana and J.M. Orensanz. - 2003 Opheliidae (Polychaeta) from the Southwestern Atlantic ocean, with the description of Travisia amadoi n. sp., Ophelina gaucha n. sp. and Ophelina alata n. sp. In: E. Sigvaldadottir, A.S.Y Mackie, G.V. Helgason, D.J. Reish, J. Svavarsson, S.A. Steingrimsson and G. Gudmundsson (eds), Advances in Polychaete Research. Proceedings of the $7^{\text {th }}$ International Polychaete Conference held in Reykjavik, Iceland, 2-6 July 2001. Hydrobiologia, 496: 75-85.

Fauchald, K. - 1977. The Polychaete Worms. Definitions and keys to the orders, families and genera. Serial Publications of the Natural History Museum of Los Angeles County. Science Series 28: $1-188$.

Fauvel, P. - 1914. Annélides polychètes non pelagiques provenant des campagnes de l'Hirondelle et de la Princesse-Alice (18851910). Résult. Camp. Scient. Prince Albert I, 46: 1-432, 31 pls.

Fauvel, P - 1941. Annélides polychètes de la Mission de Cap Horn (1882-1883). Bull. Mus. Hist. nat. Paris, sér. 2, 18: 272-298.

Fauvel, P. - 1951. Mission du batiment polaire CommandantCharcot. Recoltes faites en Tere Adélie (1950) par Paul Tchernia. Annélides polychètes. Bull. Mus. Hist. nat. Paris, sér. 2, 22: 753-773.

Hansen, G.A. - 1878. Annelider fra den norske Nordhavsexpedition i 1876. Nyt Mag. Naturv. Oslo 24(1): 1-17.

Hartman, O. - 1948. The marine annelids erected by Kinberg with notes on some other types in the Swedish State Museum. Ark. Zool. Stockholm, 42A(1): 1-137.

Hartman, O. - 1953. Non-pelagic Polychaeta of the Swedish Antarctic Expedition 1901-1903. Zoological Results Swed. Antarct. Exped. 1901-1903, 4(11): 1-83.
Hartman, O. - 1966. Polychaeta Myzostomidae and Sedentaria of Antarctica. Antarctic Res. Ser., 7: 1-158.

Hartman, O. - 1967. Polychaetous annelids collected by the USNS Eltanin and Staten Island cruises, chiefly from Antarctic seas. Allan Hancock Monogr. Mar. Biol., 2: 1-387.

Hartman, O. - 1978. Polychaeta from the Weddell Sea Quadrant, Antarctica. Antarctic Res. Ser., 26(4): 125-223.

Hartmann-Schröder, G. - 1971. Annelida, Borstenwürmer, Polychaeta. Tierwelt Deutschlands 58: 1-594.

Hartmann-Schröder,O. and P. Rosenfeldt. - 1989. Die Polychaeten der "Polarstern"-Reise ANT III/2 in die Antarktis 1984. Teil 2: Cirratulidae bis Serpulidae. Mitt. hamb. zool. Mus. Inst., 86: 65106.

Hartmann-Schröder and P. Rosenfeldt. - 1991. Die Polychaeten der "Walther Herwig"-Reise 68/1 nach Elelphant Island (Antarktis) 1985. teil 2: Acrocirridae bis Sabellidae. Mitt. hamb. zool. Mus. Inst., 88: 73-96.

Hilbig, B. - 2001. Deep-sea polychaetes in the Weddell Sea and Drake Passage: first quantitative results. Polar Biol., 24(7): 538-544.

Kinberg, J. - 1866. Annulata nova. Oefv. Vet. Akad. Stockholm, Forh., 22: 239-258.

Knox, G.A. - 1962. Antarctic polychaetes from Mawson, MacRobertson Land. Trans. Roy. Soc. N.Z. Zool. 1(27): 343348 .

Marenzeller, E. von. - 1892. Zoologische Ergebnisse der im Jahre 1889 auf Kosten der Bremer Geographischen Gesellschaft von Dr. Willy Kükenthal und Dr. Alfred Walter ausgeführten Expedition nach Ostspitzbergen. Zool. Jahrb. Jena, Abt. Syst., 6: 397-434

McIntosh, W.C. - 1885. Report on the Annelida Polychaeta collected by H.M.S. Challenger during the years 1873-76. Challenger Rep., 12: 1-554.

McIntosh, W.C. - 1908. Notes from the Gatty Marine Laboratory, St. Andrews. No. 29-3.On the same Families of Annelids in the Porcupine expeditions of 1869 and 1870. Ann.Mag. Nat. Hist., ser 8, 1: 373-387.

Monro, C.C.A. - 1930. Polychaete worms. Discovery Rep., 2: 1222.

Monro, C.C.A. - 1936. Polychaete worms. II. Discovery Rep., 12: 59-198.

Monro, C.C.A. - 1939. Polychaeta. Antarctic Research Expedition, 1929-1931. Adelaide, Australia. Reports, Ser. B (Zoology and Botany), 4(4): 89-156.

Persson, J. and F. Pleijel. - 2005. On the phylogenetic relationships of Axiokebuita, Travisia and Scalibregmatidae (Polychaeta). Zootaxa., 998: 1-14.

Rouse, G.W. - 2001. 8. Opheliidae Malmgren, 1867. In: G.W. Rouse and F. Pleijel (eds.), Polychaetes. Oxford University Press, Oxford.

Siciński, J. - 2004. Polychaetes of Antarctic sublittoral in the proglacial zone (King George Island, South Shetland Islands). Polish Polar Res., 25(1): 67-96.

Støp-Bowitz, C. - 1945. Les Ophéliens Norvégiens. Medd. zool. Mus. Oslo 52: 1-61.

Stöp-Bowitz, C. - 1948. Sur les polychetès arctiques des familles des Glycériens, Ophéliens, Scalibregmiens et des Flabelligériens. Troms $\varnothing$ Mus. Årsh. Naturh. avd. nr. 35, 66(2): $1-42$.

Tebble, N. - 1953. A review of the genus Ophelia (Polychaeta) with descriptions of new species from South African and Californian waters. Ann. Mag. Nat. Hist. Ser. 12, 6: 361-368.

Willey, A. - 1902. Report on the collections of natural history made in the Antarctic regions during the voyage of the Southern Cross. London, vol. 12, Polychaeta. pp. 262-283, pls. 41-46.

Received September 14, 2004. Accepted May 11, 2005. 